\title{
A Search for Optical Emission from Binary Black Hole Merger GW170814 with the Dark Energy Camera
}

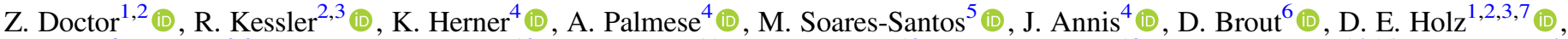
M. Sako ${ }^{6}$, A. Rest ${ }^{8,9}$ (D), P. Cowperthwaite ${ }^{10}$ (D), E. Berger ${ }^{11}$ (D), R. J. Foley ${ }^{12}$, C. J. Conselice ${ }^{13}$ (D) M. S. S. Gill ${ }^{14,15}$ (iD) S. Allam ${ }^{4}$, E. Balbinot ${ }^{16}$, R. E. Butler ${ }^{17}$ (D), H.-Y. Chen ${ }^{18}$ (D), R. Chornock ${ }^{19}$ (D), E. Cook $^{20}$, H. T. Diehl ${ }^{4}$, B. Farr ${ }^{21}$ (D), W. Fong ${ }^{22}$ (iD),

J. Frieman ${ }^{2,4}$, C. Fryer $^{23}$ (D), J. García-Bellido ${ }^{24}$ (D), R. Margutti ${ }^{22}$ (D), J. L. Marshall ${ }^{20}$ (D), T. Matheson ${ }^{25}$ (D), B. D. Metzger ${ }^{26}$ (iD), M. Nicholl ${ }^{27}$ (D) , F. Paz-Chinchón ${ }^{28}$ (D), S. Salim ${ }^{17}$ (D) M. Sauseda ${ }^{29}$, L. F. Secco ${ }^{6}$ (D) R. C. Smith ${ }^{30}$, N. Smith ${ }^{31}$ (D), A. K. Vivas ${ }^{30}$ (D), D. L. Tucker ${ }^{4}$, T. M. C. Abbott $^{30}$ (D), S. Avila ${ }^{32}$, K. Bechtol ${ }^{33,34}$, E. Bertin ${ }^{35,36}$, D. Brooks ${ }^{37}$ (D) E. Buckley-Geer ${ }^{4}$,

D. L. Burke ${ }^{14,15}$ (I) , A. Carnero Rosell ${ }^{38,39}$, M. Carrasco Kind ${ }^{40,41}$ (D), J. Carretero ${ }^{42}$, F. J. Castander ${ }^{43,44}$ (ID), C. B. D’Andrea ${ }^{6}$, L. N. da Costa ${ }^{39,45}$, J. De Vicente ${ }^{38}$, S. Desai ${ }^{46}$ (D) P. Doel $^{37}$, B. Flaugher ${ }^{4}$, P. Fosalba ${ }^{43,44}$, E. Gaztanaga ${ }^{43,44}$, D. W. Gerdes ${ }^{47,48}$ (iD), D. A. Goldstein ${ }^{49}$ (D) D. Gruen ${ }^{14,15}$ (D), R. A. Gruendl ${ }^{40,41}$ (iD) G. Gutierrez ${ }^{4}$, W. G. Hartley ${ }^{37,50}$, D. L. Hollowood ${ }^{12}$ (iD), K. Honscheid ${ }^{51,52}$, B. Hoyle ${ }^{53,54}$, D. J. James ${ }^{55}$ (D), T. Jeltema ${ }^{12}$, S. Kent ${ }^{2,4}$, K. Kuehn ${ }^{56}$ (D), N. Kuropatkin ${ }^{4}$, O. Lahav ${ }^{37}$, M. Lima ${ }^{39,57}$, M. A. G. Maia ${ }^{39,45}$, M. March ${ }^{6}$, F. Menanteau ${ }^{40,41}$, C. J. Miller ${ }^{47,48}$, R. Miquel ${ }^{42,58}$ (ID) E. Neilsen ${ }^{4}$, B. Nord ${ }^{4}$ (D), R. L. C. Ogando ${ }^{39,45}$ (D), A. A. Plazas ${ }^{59,60}$ (D), A. Roodman ${ }^{14,15}$ (D), E. Sanchez ${ }^{38}$ (D), V. Scarpine ${ }^{4}$, R. Schindler ${ }^{14}$, M. Schubnell ${ }^{48}$, S. Serrano ${ }^{43,44}$, I. Sevilla-Noarbe ${ }^{38}$, M. Smith ${ }^{61}$ (D) F. Sobreira ${ }^{39,62}$ (D) E. Suchyta ${ }^{63}$, M. E. C. Swanson ${ }^{41}$ (D), G. Tarle ${ }^{48}$ (iD), D. Thomas ${ }^{32}$, A. R. Walker ${ }^{30}\left(\mathbb{D}\right.$, and W. Wester ${ }^{4}$

(DES Collaboration)

${ }^{1}$ Department of Physics, University of Chicago, Chicago, IL 60637, USA

${ }^{2}$ Kavli Institute for Cosmological Physics, University of Chicago, Chicago, IL 60637, USA

${ }^{3}$ Department of Astronomy and Astrophysics, University of Chicago, Chicago, IL 60637, USA

${ }^{4}$ Fermi National Accelerator Laboratory, P.O. Box 500, Batavia, IL 60510, USA

${ }^{5}$ Brandeis University, Physics Department, 415 South Street, Waltham, MA 02453, USA

${ }^{6}$ Department of Physics and Astronomy, University of Pennsylvania, Philadelphia, PA 19104, USA

${ }^{7}$ Enrico Fermi Institute, University of Chicago, Chicago, IL 60637, USA

${ }^{8}$ Department of Physics and Astronomy, The Johns Hopkins University, 3400 North Charles Street, Baltimore, MD 21218, USA

${ }^{9}$ Space Telescope Science Institute, 3700 San Martin Drive, Baltimore, MD 21218, USA

${ }^{10}$ Carnegie Observatories, 813 Santa Barbara Street, Pasadena, CA 91104, USA

${ }^{11}$ Harvard-Smithsonian Center for Astrophysics, 60 Garden Street, Cambridge, MA 02138, USA

${ }^{12}$ Department of Astronomy and Astrophysics, University of California, Santa Cruz, CA 95064, USA

${ }^{13}$ Centre for Astronomy and Particle Theory, School of Physics \& Astronomy, University of Nottingham, Nottingham NG7 2RD, UK

${ }^{14}$ SLAC National Accelerator Laboratory, Menlo Park, CA 94025, USA

${ }^{15}$ Kavli Institute for Particle Astrophysics \& Cosmology, P.O. Box 2450, Stanford University, Stanford, CA 94305, USA

${ }^{16}$ Kapteyn Astronomical Institute, University of Groningen, Landleven 12, 9747 AD Groningen, The Netherlands

${ }^{17}$ Department of Astronomy, Indiana University, 727 East Third Street, Bloomington, IN 47405, USA

${ }^{18}$ Black Hole Initiative, Harvard University, Cambridge, MA 02138, USA

${ }^{19}$ Astrophysical Institute, Department of Physics and Astronomy, 251B Clippinger Lab, Ohio University, Athens, OH 45701, USA

${ }^{20}$ George P. and Cynthia Woods Mitchell Institute for Fundamental Physics and Astronomy, and Department of Physics and Astronomy, Texas A\&M University, College Station, TX 77843, USA

${ }^{21}$ Department of Physics, University of Oregon, Eugene, OR 97403, USA

${ }^{22}$ Center for Interdisciplinary Exploration and Research in Astrophysics (CIERA) and Department of Physics and Astronomy, Northwestern University, Evanston, IL 60208, USA

${ }^{23}$ Center for Theoretical Astrophysics, Los Alamos National Laboratory, Los Alamos, NM 87545, USA

${ }_{24}^{24}$ Instituto de Fisica Teorica UAM/CSIC, Universidad Autonoma de Madrid, E-28049 Madrid, Spain

${ }^{25}$ National Optical Astronomy Observatory, 950 North Cherry Avenue, Tucson, AZ 85719, USA

${ }_{27}^{26}$ Department of Physics and Columbia Astrophysics Laboratory, Columbia University, New York, NY 10027, USA

${ }^{27}$ Institute for Astronomy, University of Edinburgh, Royal Observatory, Blackford Hill, Edinburgh EH9 3HJ, UK

${ }^{28}$ National Center for Supercomputing Applications, University of Illinois at Urbana-Champaign, 1205 West Clark Street, Urbana, IL 61801, USA

${ }^{29}$ Department of Physics and Astronomy, Texas A\&M University, College Station, TX 77843, USA

${ }^{30}$ Cerro Tololo Inter-American Observatory, National Optical Astronomy Observatory, Casilla 603, La Serena, Chile

${ }^{31}$ Steward Observatory, University of Arizona, 933 North Cherry Avenue, Tucson, AZ 85721, USA

${ }^{32}$ Institute of Cosmology and Gravitation, University of Portsmouth, Portsmouth PO1 3FX, UK

${ }^{33}$ LSST, 933 North Cherry Avenue, Tucson, AZ 85721, USA

${ }^{34}$ Physics Department, 2320 Chamberlin Hall, University of Wisconsin-Madison, 1150 University Avenue, Madison, WI 53706-1390, USA

${ }^{35}$ CNRS, UMR 7095, Institut d'Astrophysique de Paris, F-75014, Paris, France

${ }^{36}$ Sorbonne Universités, UPMC Univ Paris 06, UMR 7095, Institut d'Astrophysique de Paris, F-75014, Paris, France

${ }^{37}$ Department of Physics \& Astronomy, University College London, Gower Street, London WC1E 6BT, UK

${ }^{38}$ Centro de Investigaciones Energéticas, Medioambientales y Tecnológicas (CIEMAT), Madrid, Spain

${ }^{39}$ Laboratório Interinstitucional de e-Astronomia-LIneA, Rua Gal. José Cristino 77, Rio de Janeiro, RJ-20921-400, Brazil

${ }^{40}$ Department of Astronomy, University of Illinois at Urbana-Champaign, 1002 West Green Street, Urbana, IL 61801, USA

${ }^{41}$ National Center for Supercomputing Applications, 1205 West Clark Street, Urbana, IL 61801, USA

${ }^{42}$ Institut de Física d'Altes Energies (IFAE), The Barcelona Institute of Science and Technology, Campus UAB, E-08193 Bellaterra (Barcelona) Spain

${ }^{43}$ Institut d'Estudis Espacials de Catalunya (IEEC), E-08034 Barcelona, Spain

${ }^{44}$ Institute of Space Sciences (ICE, CSIC), Campus UAB, Carrer de Can Magrans, s/n, E-08193 Barcelona, Spain

${ }^{45}$ Observatório Nacional, Rua Gal. José Cristino 77, Rio de Janeiro, RJ-20921-400, Brazil

${ }^{46}$ Department of Physics, IIT Hyderabad, Kandi, Telangana 502285, India

${ }^{47}$ Department of Astronomy, University of Michigan, Ann Arbor, MI 48109, USA 


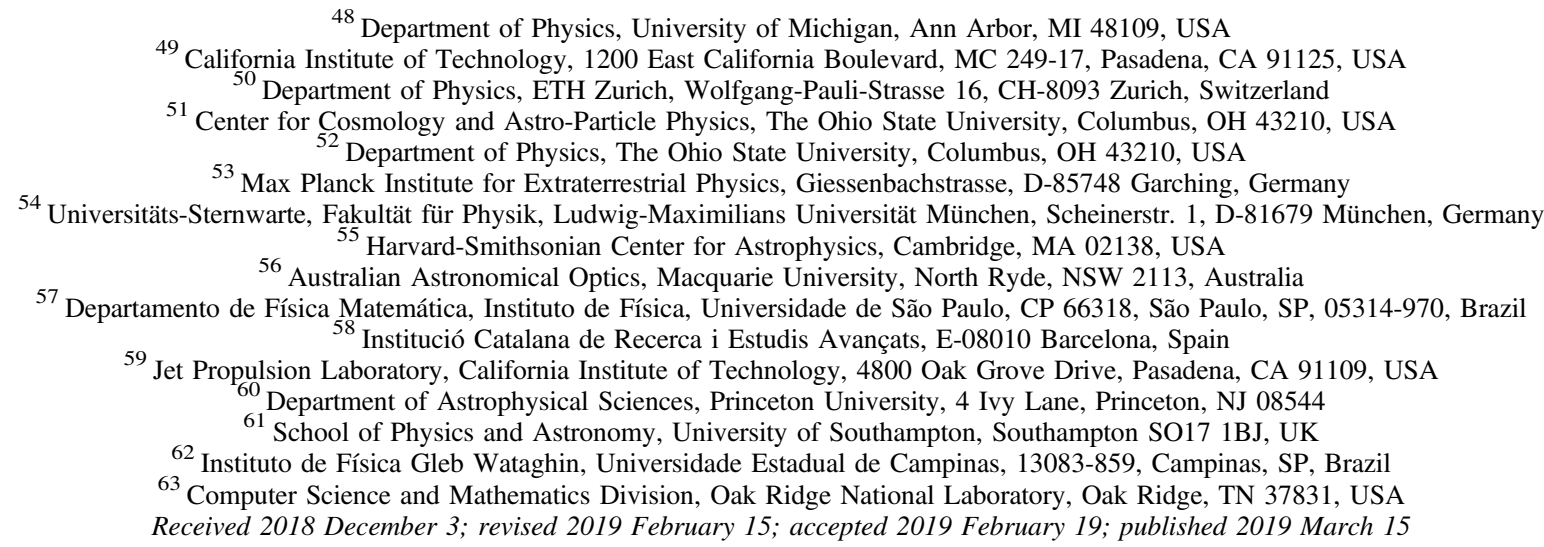

\begin{abstract}
Binary black hole (BBH) mergers found by the Laser Interferometer Gravitational-Wave Observatory (LIGO) and Virgo detectors are of immense scientific interest to the astrophysics community, but are considered unlikely to be sources of electromagnetic emission. To test whether they have rapidly fading optical counterparts, we used the Dark Energy Camera to perform an $i$-band search for the BBH merger GW170814, the first gravitational wave (GW) detected by three interferometers. The $87 \mathrm{deg}^{2}$ localization region (at $90 \%$ confidence) centered in the Dark Energy Survey (DES) footprint enabled us to image 86\% of the probable sky area to a depth of $i \sim 23$ mag and provide the most comprehensive data set to search for electromagnetic (EM) emission from BBH mergers. To identify candidates, we perform difference imaging with our search images and with templates from pre-existing Dark Energy Survey (DES) images. The analysis strategy and selection requirements were designed to remove supernovae and to identify transients that decline in the first two epochs. We find two candidates, each of which is spatially coincident with a star or a high-redshift galaxy in the DES catalogs, and they are thus unlikely to be associated with GW170814. Our search finds no candidates associated with GW170814, disfavoring rapidly declining optical emission from BBH mergers brighter than $i \sim 23$ mag $\left(L_{\text {optical }} \sim 5 \times 10^{41} \mathrm{erg} \mathrm{s}^{-1}\right) 1-2$ days after coalescence. In terms of GW sky map coverage, this is the most complete search for optical counterparts to BBH mergers to date.
\end{abstract}

Key words: gravitational waves - stars: black holes - surveys - techniques: photometric

\section{Introduction}

Since the first binary black hole (BBH) merger detection in 2015 September (Abbott et al. 2016a), mergers of two black holes have become a mainstay of gravitational-wave $(\mathrm{GW})$ astrophysics. The first five observed BBHs, found only by the Hanford and Livingston Laser Interferometer GravitationalWave Observatory (LIGO) detectors, offered significant astrophysical insight into the $\mathrm{BBH}$ mass distribution and event rates (Abbott et al. 2016a, 2016b, 2016c, 2016d, 2017b, 2017c). For electromagnetic (EM) follow-up, however, the two LIGO detectors alone place poor constraints on the sky position, typically a few hundred $\mathrm{deg}^{2}$.

To date, no compelling optical counterparts to BBH mergers have been identified. However, Stalder et al. (2017) found optical candidate ATLAS17aeu in their follow-up of GW170104 and hypothesize a chance coincidence. Additionally, a weak gamma-ray burst in coincidence with GW150914 was reported in Connaughton et al. (2016), but its association with GW150914 is still under dispute. There are three (not mutually exclusive) reasons for non-detections: (1) the probable sky regions of previous $\mathrm{BBH}$ detections were not searched comprehensively, (2) the BBH emission could not be identified or distinguished from background transients, and/or (3) optical emission from $\mathrm{BBH}$ mergers is non-existent or below the detectable threshold at the times of the existing observations. Theoretical models have been proposed that could produce EM signals (e.g., Loeb 2016; Perna et al. 2016; de
Mink \& King 2017; Stone et al. 2017; McKernan et al. 2018), but these models are highly speculative. With little theoretical guidance, there is a need for more complete searches for BBH EM emission while also controlling false-positive event rates. Detection of BBH EM counterparts would be of immense scientific value (e.g., Phinney 2009), as it could constrain the formation environments of BBHs, the behavior of matter in strong field gravity, and cosmological parameters such as the Hubble constant. $^{64}$

Thus far, a number of optical follow-up campaigns have been conducted to search for BBH counterparts (e.g., Cowperthwaite et al. 2016; Morokuma et al. 2016; Smartt et al. 2016a, 2016b; Soares-Santos et al. 2016; Lipunov et al. 2017; Stalder et al. 2017; Yoshida et al. 2017; Smith et al. 2018). However, the large probable sky areas of the "double-coincident" LIGO detections (Hanford and Livingston detectors only) curtailed searches for EM counterparts from BBH mergers. For example, Soares-Santos et al. (2016) observed $102 \mathrm{deg}^{2}$ of the GW150914 high-probability sky region with the optical imager Dark Energy Camera, (DECam; Flaugher et al. 2015), corresponding to 38\% of the initial LIGO sky map probability. After accounting for the lack of existing images (templates) for difference imaging, a shift in the sky map in a reanalysis of LIGO data, and other efficiency

\footnotetext{
${ }^{64}$ Even without an optical counterpart to a $\mathrm{BBH}$, it is possible to measure the Hubble constant with a BBH GW sky map and galaxy catalog as in e.g., Schutz (1986), Chen et al. (2018), Fishbach et al. (2019), and The DES Collaboration et al. (2019).
} 
losses, only 3\% of the probable GW150914 sky area was searched and analyzed. Similarly, the DECam follow-up campaign of GW151226 reported in Cowperthwaite et al. (2016) covered $29 \mathrm{deg}^{2}$, just $\sim 2 \%$ of the final GW151226 highprobability region. In contrast, with the three-detector network including the Virgo interferometer, the smaller $28 \mathrm{deg}^{2} 90 \%$ localization region of neutron-star merger GW170817 enabled $81 \%$ DECam coverage of the final LIGO-Virgo sky map and identification of the EM counterpart (Abbott et al. 2017a; Soares-Santos et al. 2017). These searches were all performed in the $i$ - and $z$-bands, requiring two tilings of the search area. We note that these DECam searches attempted to tile maximal sky map probability, but for the nearby events such as GW170817, targeting based on galaxy catalogs can be successful (e.g., Arcavi et al. 2017; Coulter et al. 2017; Valenti et al. 2017).

On 2017 August 14, the LIGO-Virgo Collaboration (LVC), with the addition of the Virgo detector, made the first "triplecoincident" detection of GWs from a BBH event, GW170814, and provided a much tighter constraint on the sky position of the source than those of previous BBH detections (Abbott et al. 2017d; The LIGO Scientific Collaboration \& the Virgo Collaboration 2018). The detection of GW170814, with its $87 \mathrm{deg}^{2} 90 \%$-localization region, enabled our team to perform a comprehensive search of the sky area for BBH merger optical counterparts and significantly improve our sensitivity to $\mathrm{BBH}$ merger EM emission models.

We report on our search for optical counterparts to GW170814 using DECam. In Section 2, we describe the parameters and cadence of our follow-up observations, which extended to 12 days after the GW170814 trigger and covered $225 \mathrm{deg}^{2}$. Section 3 describes the analysis. Finally, Section 4 presents the results of the analysis, which we then comment on in Sections 5 and 6.

\section{Search and Light Curves}

On 2017 August 14 at 10:30:43 UTC, the LVC reported a signal consistent with the inspiral and merger of two black holes of masses $30.5_{-3.0}^{+5.7} M_{\odot}$ and $25.3_{-4.2}^{+2.8} M_{\odot}$ at a luminosity distance of $540_{-210}^{+130} \mathrm{Mpc}$ and redshift ${ }^{65} z=0.12_{-.04}^{+.03}$ (Abbott et al. 2017d). LIGO and Virgo sent out a Bayestar sky map $2 \mathrm{hr}$ after the trigger (Singer \& Price 2016; LIGO-Virgo Collaboration 2017a) and we captured our first DECam image of the probability region at 06:00 UTC on August 15, $19.5 \mathrm{hr}$ after the GW detection. DECam is an optical imager, installed on the Blanco $4 \mathrm{~m}$ telescope at the Cerro Tololo Inter-American Observatory. It has a $3 \mathrm{deg}^{2}$ field of view and is equipped with several broadband optical/near-infrared (NIR) filters $(u, g, r, i$, $z, Y, V R)$, making it well suited to search for faint transients over large sky areas (Flaugher et al. 2015).

We imaged the high-probability area of the Bayestar sky map in the $i$-band with $90 \mathrm{~s}$ exposures, corresponding to a $5 \sigma$ point-source depth of $\approx 23 \mathrm{mag}$. Our strategy of imaging the most probable regions was similar to that used in Soares-Santos et al. (2016) and Cowperthwaite et al. (2016) (who surveyed in the $i$ - and $z$-bands), but in order to maximize the sky area coverage we only surveyed in the $i$-band. Our search covered $225 \mathrm{deg}^{2}$, corresponding to $90 \%$ of the initial Bayestar map, $90 \%$ of the LALInference sky map that was released with the original GW170814 LIGO-Virgo publication (Abbott et al. $2017 \mathrm{~d}$ ), and $86 \%$ of the final map from the O1-O2 GW catalog

\footnotetext{
65 Assuming cosmology of Planck Collaboration (2016).
}

(The LIGO Scientific Collaboration \& the Virgo Collaboration 2018). These estimates account for chip gaps on the camera but not masking of bright stars. A preliminary LALInference map accounting for calibration uncertainties was sent after our first night of observations, causing a shift in the search region on the second night of observations and onward (LIGO-Virgo Collaboration 2017b). Figure 1 shows our tiling over the LIGO-Virgo sky maps. Observations of the region of interest were taken in epochs that began roughly $0.8,1.8,2.8,5.8,8.8$, $9.8,10.8$, and 11.8 days after the GW event, and each epoch's tiling spanned about $4 \mathrm{hr}$. The $5 \sigma$ limiting point-source magnitude in the $i$-band was approximately 24 over all the tiles on the first night of observations. The first DECam image was taken on 2017 August 15 at 06:05:31 UTC. Our cadence was chosen to have a dense sampling in time, but observing conditions and follow up of GW170817 introduced larger gaps between the third and eighth nights.

We processed the images from our search using the Dark Energy Survey's (DES) transient detection pipeline as in Soares-Santos et al. (2016) and K. Herner et al. (2019, in preparation). The pipeline consists of a single-epoch processing stage (Bernstein et al. 2017; Morganson et al. 2018) followed by a stage that takes the difference of search images and template images to identify sources with fluctuating brightness (DiffImg; Kessler et al. 2015). Template images were available from existing DES data as the LIGO-Virgo sky maps were contained in the DES footprint. The sources detected in the pipeline are used to generate candidate light curves: a candidate requires at least two detections by the pipeline, and for each candidate a light curve is constructed from a pointspread function (PSF)-fitted flux at each observation. The pipeline also removes persistent point sources in the DES Y1 catalog that are brighter than $20.5 \mathrm{mag}$ in any band.

We split the data into two samples because of a shift in the GW sky map after the first night of observations. This shift prompted a change in the patch of sky that we targeted, creating inhomogeneity in the data sample as the cadence of observations was not uniform over the full area that we imaged. The red and orange "hexes" in Figure 1 show which fields were observed the first night versus only on later nights, respectively. The first data set, D1, includes the $N_{\mathrm{D} 1}=42368$ candidates that were first observed $\sim 0.8$ days after the $\mathrm{GW}$ trigger when we were targeting the Bayestar sky map. D2 contains the $N_{\mathrm{D} 2}=17192$ candidates observed for the first time after acquiring the preliminary LALInference sky map. Over the full GW170814 follow-up campaign, the median number of observations per candidate is eight and five for D1 and $\mathrm{D} 2$, respectively.

\section{Analysis}

To identify candidates of interest, we apply selection requirements (or "cuts") to the full set of candidates produced by DiffImg. We present these criteria in Section 3.2 and have chosen them to (a) minimize contamination from both astrophysical transients such as supernovae ( $\mathrm{SNe}$ ) and asteroids as well as artifacts in the data, and (b) identify "fast transients" that quickly decline after the merger. SNANA simulations (Kessler et al. 2009) of Type Ia and core-collapse SN light curves (using the SALT-II Ia light curve model of Guy et al. (2010) and Ibc, IIp, IIn core-collapse templates from Kessler et al. 2010) provide guidance on cuts to remove SNe. A full optimization and exploration of the cuts is not explored here 


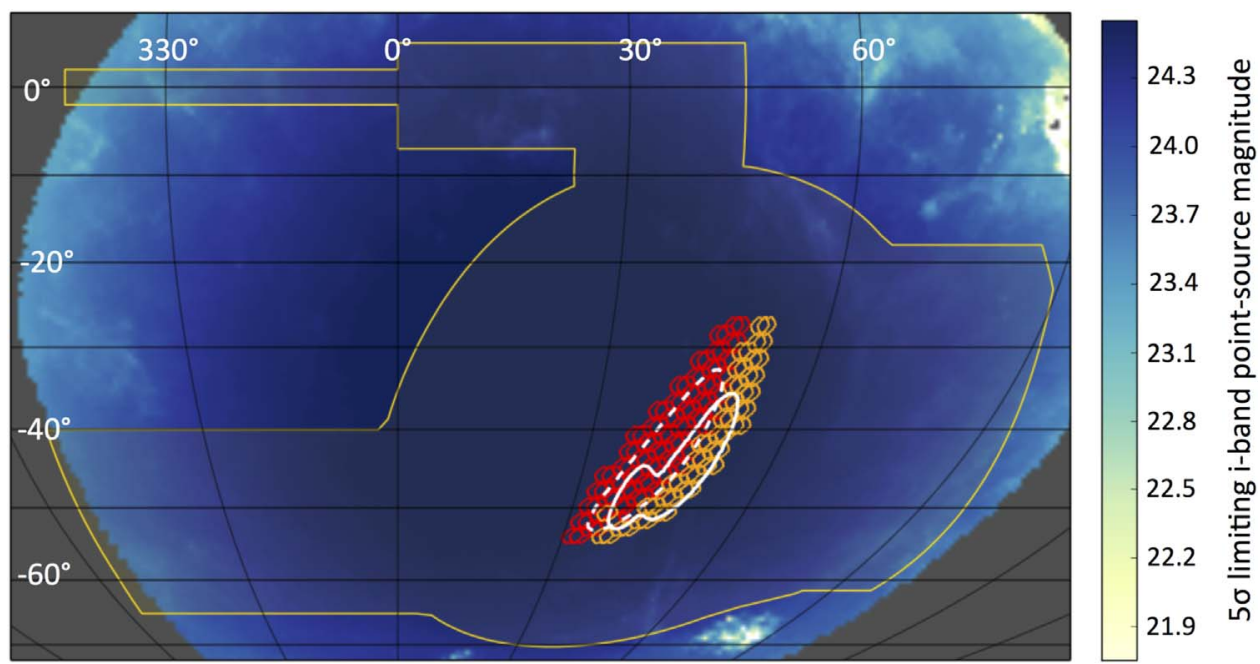

Figure 1. Dithered tiling performed for GW170814 overlaid on the GW170814 90\%-confidence sky area contours. The red hexes show the individual pointings that were performed in our search on the first night of observations. The orange hexes represent the tiles that were not observed until the second night or later due to the sky map change. The white dotted contour shows the initial Bayestar map, and the solid white contour represents the final sky map from the LIGO-Virgo O1-O2 GW catalog. The region enclosed by the yellow contour corresponds to the Dark Energy Survey (DES) footprint, and the background color shows the estimated $5 \sigma$ pointsource limiting magnitude for a $90 \mathrm{~s}$ exposure which accounts for air mass and dust extinction (see Neilsen et al. 2016).

and is left for future analyses. We choose these cuts using a control sample of candidates that are away from the highest probability regions of the LALInference sky map, as described in Section 3.1. The number of candidates remaining in the control sample after applying cuts is used to infer the number of candidates expected in the full sample. This inference is detailed in Section 3.3.

Our analysis also makes use of two auxiliary tools. The first is a machine-learning (ML) algorithm, autoScan, trained on DES difference images that produces a score for each difference-imaging detection between 0 and 1 , with high scores corresponding to high-confidence point-source-like sources (Goldstein et al. 2015). The second is a set of objectfitting algorithms that classify persistent DES sources as galaxies or stars depending on the sources' spatial extent (Drlica-Wagner et al. 2018; Sevilla-Noarbe et al. 2018). We use these tools to identify high-confidence point-source detections in our search and to match these detections with stars and galaxies.

\subsection{Control Sample}

To reduce potential bias in tuning the analysis cuts to reject all events, the cuts are optimized on a control sample. The control sample comprises a random third of all DiffImg candidates, and candidates within $4.5 \mathrm{deg}$ of the maximum a posteriori point of the Abbott et al. (2017d) LALInference sky map are excluded. There is an $\sim 8 \%$ and $\sim 10 \%$ chance that the true location of GW170814 is in the control region based on the Abbott et al. (2017d) and The LIGO Scientific Collaboration \& the Virgo Collaboration (2018) sky maps, respectively.

As with the full data set, we split our control sample into two subsamples. The first subsample $\mathrm{C} 1$ (with $N_{\mathrm{C} 1}=12381$ candidates) comprises the control candidates in D1. The second subsample $\mathrm{C} 2$ contains the control candidates $\left(N_{\mathrm{C} 2}=3867\right.$ candidates $)$ in $\mathrm{D} 2$. We apply the cuts in Section 3.2 to the two control subsamples and record the sets
Table 1

The Number of Candidates in the Two Subsets of Full (D), Control (C), and Blinded (B) Samples

\begin{tabular}{lllr}
\hline \hline$N_{\text {D1 }}$ & 42,368 & $N_{\text {D2 }}$ & 17,192 \\
$N_{\mathrm{C} 1}$ & 12,381 & $N_{\mathrm{C} 2}$ & 3867 \\
$N_{\text {B } 1}$ & 29,987 & $N_{\text {B2 }}$ & 13,325 \\
\hline
\end{tabular}

of candidates $\mathrm{c} 1$ and $\mathrm{c} 2$ (with $N_{\mathrm{c} 1}$ and $N_{\mathrm{c} 2}$ candidates, respectively) passing cuts out of the totals.

The remaining data (which we call the blinded sample) is similarly split into two subsamples B1 and B2 for events first observed when targeting the Bayestar map and LALInference map, respectively. In total, Subsample B1 contains $N_{\mathrm{B} 1}=29987$ candidates and B2 contains $N_{\mathrm{B} 2}=13325$ candidates. Because $\mathrm{B} 1, \mathrm{~B} 2, \mathrm{C} 1$, and $\mathrm{C} 2$ are mutually exclusive, we have $N_{\mathrm{D} 1}=N_{\mathrm{B} 1}+N_{\mathrm{C} 1}$ and $N_{\mathrm{D} 2}=N_{\mathrm{B} 2}+N_{\mathrm{C} 2}$. Table 1 summarizes the numbers of candidates in each subsample.

\subsection{Selection Requirements}

Below we list the cuts applied to the candidates.

1. Raw sample: all candidates produced by DiffImg.

2. First-epoch ML $>0.7$ : using the autoScan ML score $(0<\mathrm{ML}<1)$ that was trained with DES data (Goldstein et al. 2015) to remove non-point-source-like detections, we require $\mathrm{ML}>0.7$ for the first observation. This cut eliminates image artifacts that arise in the difference imaging. For reference, the DES Supernova program requires $\mathrm{ML}>0.5$, but for two separate detections of a candidate rather than just one detection. Our requirement is more stringent because we are looking for rapidly fading sources, and therefore only cut on the first-epoch ML. Our stricter $\mathrm{ML}>0.7$ requirement lowers the numbers of single-epoch false positives by a factor of $\sim 2$ compared with $\mathrm{ML}>0.5$, while lowering the efficiency by only a few percent at signal-to-noise ratio (S/N) 10 (Goldstein et al. 2015). 
3. Host galaxy $z<0.3$ : using galaxies from the DES Y3 Gold catalog, a candidate is matched to a host galaxy if it is within four times the directional light radius of the galaxy (Gupta et al. 2016). The directional light radius is the radius of a potential host galaxy in the direction of the candidate transient and is dependent on the survey. Each galaxy is also fit with a Directional Neighborhood Fitting (DNF) photometric redshift $z_{\mathrm{DNF}}$ with uncertainty $\Delta z_{\mathrm{DNF}}$ (De Vicente et al. 2016). If the candidate is matched to a galaxy and the best-match galaxy satisfies $z_{\mathrm{DNF}}-$ $\Delta z_{\mathrm{DNF}}>0.3$, the candidate is removed from the sample. This cut removes events that are clearly associated with galaxies beyond the estimated GW redshift of $z=$ $0.12_{-.04}^{+.03}$.

4. Second observation $S / N \geqslant 2$ : the candidate must have a measured $\mathrm{S} / \mathrm{N}$ of at least 2 on the second observation. Measurements within one hour of each other are not considered separate observations for this cut. This cut rejects asteroids and difference imaging artifacts.

5. Greater than $2 \sigma$ decline: there must be a $>2 \sigma$ decline in the flux between the first and second epochs when a candidate was observed. A similar cut was implemented in Soares-Santos et al. (2016) and Cowperthwaite et al. (2016). $\sigma$ is the quadrature sum of the flux errors on the two epochs. If multiple measurements of a candidate were taken in the same epoch (i.e., in the same night), we use the first measurement of the epoch. If we did not observe the candidate on the second epoch, it is removed from the sample. We note that the effect of this cut depends sensitively on the observational choices of the follow-up campaign, not just the astrophysics of the potential EM source.

6. $N_{\text {obs }} \geqslant 4$ : to ensure that we can examine each candidate's light curve over a broad portion of the follow-up campaign, the candidate must have been observed at least $N_{\text {obs }}=4$ times, regardless of $\mathrm{S} / \mathrm{N}$.

7. Late-time $\mathrm{S} / \mathrm{N}<6$ : after one week from the $\mathrm{GW}$ event, the $\mathrm{S} / \mathrm{N}$ of all observations of a candidate must be less than 6 . This requirement removes objects that are bright at late times such as $\mathrm{SNe}$ and variable stars.

8. No late-time brightening: to isolate fading transients, we require that after $48 \mathrm{hr}$ from the $\mathrm{GW}$ event, there is no increase in flux of the candidate greater than $3 \sigma$, where $\sigma$ is the quadrature sum of uncertainties on adjacent flux measurements.

9. Visual inspection: subtracted image stamps identified as artifacts (e.g., cosmic rays) are removed from the sample.

After applying these cuts to the control sample, $N_{\mathrm{c} 1}=1$ and $N_{\mathrm{c} 2}=0$ candidates remain.

\subsection{Expectation of Number of Candidates in Full Sample}

Given $N_{\mathrm{c} 1}$ and $N_{\mathrm{c} 2}$ out of $N_{\mathrm{C} 1}$ and $N_{\mathrm{C} 2}$ candidates passing in the control fields, respectively, we expect $\left\langle N_{\mathrm{b} 1}+N_{\mathrm{b} 2}\right\rangle=$ $N_{\mathrm{c} 1} N_{\mathrm{B} 1} / N_{\mathrm{C} 1}+N_{\mathrm{c} 2} N_{\mathrm{B} 2} / N_{\mathrm{C} 2}=2.4$ events in $\mathrm{B} 1+\mathrm{B} 2$, which we interpret as the mean of a Poisson distribution. This interpretation does not account for small differences in Milky Way reddening and stellar density over the search region. In Section 4 , we analyze the blinded sample and compare our expectations to the number of candidates passing the cuts.
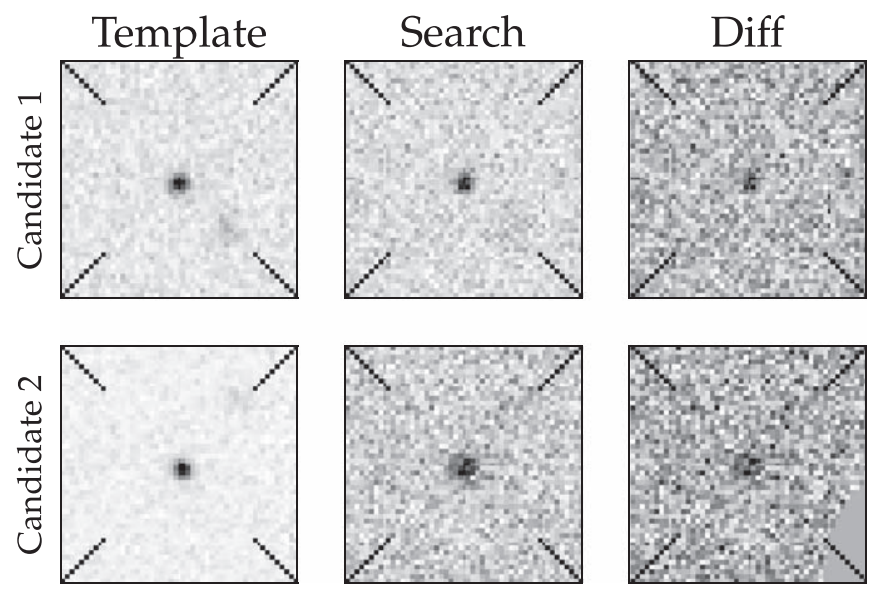

Figure 2. Template, search, and difference image stamps for candidates passing cuts. The top row shows the $i$-band images for Candidate 1 , and the bottom for Candidate 2. The search and difference images are from the the first epoch of observations of the candidate. Each stamp is 13 !! $2 \times 13$ !! 2 .

Table 2

Top: Candidates Remaining in the Full Data Sample after Applying Cuts

\begin{tabular}{|c|c|c|c|c|}
\hline \multicolumn{2}{|l|}{ Cuts } & $N_{\text {seq }}{ }^{\mathrm{a}}$ & $N_{\text {only }}{ }^{\mathrm{b}}$ & $N_{\mathrm{LO}}^{\mathrm{c}}$ \\
\hline \multicolumn{2}{|c|}{ 1. Raw sample } & 59,560 & $\cdots$ & $\cdots$ \\
\hline \multicolumn{2}{|c|}{ 2. First epoch ML $>0.7$} & 1206 & 1206 & 258 \\
\hline \multicolumn{2}{|c|}{ 3. Unmatched or host $z<0.30$} & 730 & 31,119 & 8 \\
\hline \multicolumn{2}{|c|}{ 4. Second obs. $S / N \geqslant 2.0$} & 663 & 44,181 & 4 \\
\hline \multicolumn{2}{|c|}{ 5. $>2.0$ sigma decline } & 45 & 5570 & 65 \\
\hline \multicolumn{2}{|c|}{ 6. $N_{\text {obs }} \geqslant 4$} & 31 & 50,029 & 2 \\
\hline \multicolumn{2}{|c|}{ 7. Late-time $\mathrm{S} / \mathrm{N}<6$} & 4 & 27,571 & 21 \\
\hline \multicolumn{2}{|c|}{ 8. No late-time brightening } & 2 & 36,499 & 4 \\
\hline \multicolumn{2}{|c|}{ 9. Visual inspection } & 2 & $\cdots$ & 2 \\
\hline Candidate \# & R.A. & & Decl. & $m_{i}$ \\
\hline$\overline{1}$ & 42.35047 & & -40.32632 & 22.5 \\
\hline 2 & $47^{\circ} 63365$ & & -36.36045 & 21.9 \\
\hline
\end{tabular}

Notes. Bottom: sky coordinates and initial $i$-band magnitude $m_{i}$ of the two candidates passing all cuts.

${ }^{a}$ Number of candidates remaining after applying each cut sequentially.

${ }^{\mathrm{b}}$ Number of candidates after applying an individual cut.

c Number of candidates if a cut is "left out" but all the rest are applied.

\section{Results}

Table 2 shows the effect of the cuts on the full sample, which includes the control sample. It also shows the initial $i$-band magnitudes and sky positions for the events passing all cuts. After analyzing the blinded sample, one more candidate is found, leaving a total of two candidates passing cuts in the control and blinded samples, with ID numbers 1 and 2, respectively. Finding one candidate passing cuts in the blinded sample is consistent with the 2.4 expected background events derived from the control sample presented in Section 3.3. The light curves for both events and their sky positions are shown in Figure 3.

Upon visual inspection of the two candidates, neither is an obvious subtraction artifact or cosmic ray. Here we do not show examples of subtraction artifacts and cosmic rays that would be cut by visual inspection, because visual inspection did not end up removing any candidates in this analysis. 

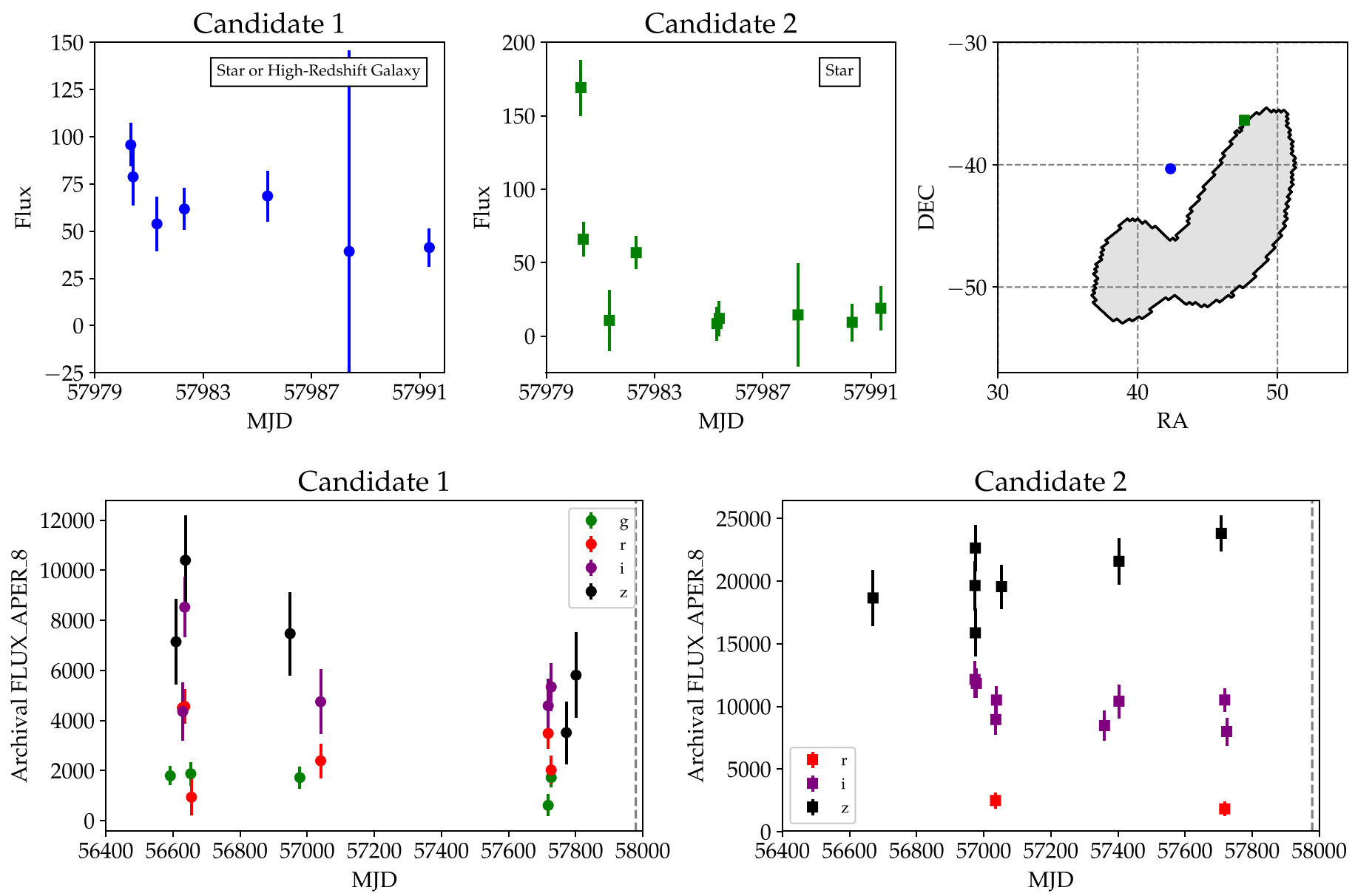

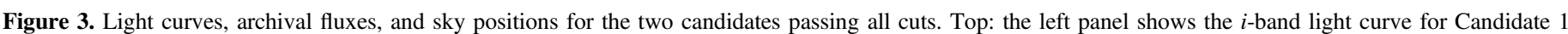

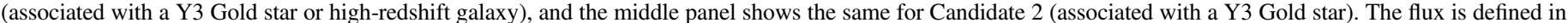

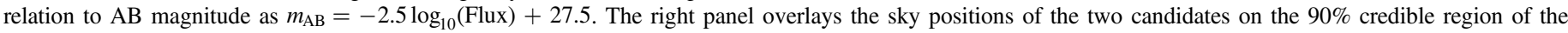

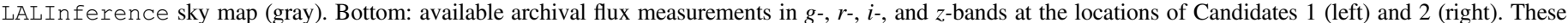

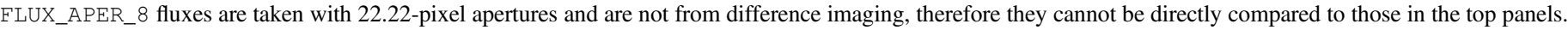
The vertical, gray, dashed line on the far right of the two plots indicates the GW170814 merger time.

However, the template images for both candidates contain a bright source at the position of the candidates. The template, search, and difference images from the first epoch of observations of each candidate are shown in Figure 2.

A deeper search through the DES high-quality object catalog ("Y3 Gold") reveals that Candidate 1 is associated with an object that is classified as either a galaxy at $z \sim 0.9$, or a star, depending on the classifier used. A multi-epoch, multi-objectfitting algorithm classifies the object's PSF as a candidate star, whereas the single-object fit categorizes the object as a galaxy (Drlica-Wagner et al. 2018; Sevilla-Noarbe et al. 2018). Notably, the object is too faint to meet the brightness cutoff for inclusion in our star veto catalog and it is not vetoed by our host galaxy redshift cut (cut 3) because we only include the highest confidence galaxies in the host-galaxy matching. Fitting each band to a constant flux for all archival observations of the object (DES Years $1-4$ ) results in a $\chi^{2} /$ DOF of $48.6 / 17=2.9$ and $p$-value $p\left(\chi^{2} \geqslant 48.6 \mid \mathrm{DOF}=17\right)=7 \times 10^{-5}$, indicating previous variability of the source. These archival fluxes are shown in Figure 3. Spectroscopic observations of this source could clarify if the object is a star or galaxy.

Candidate 2 is also associated with a DES Y3 Gold object and is classified as a star by both classifiers; constant-flux fits to archival observations yield a $\chi^{2} /$ DOF of $25.7 / 14=1.8$ and $p$-value of $p\left(\chi^{2} \geqslant 25.7 \mid \mathrm{DOF}=14\right)=0.03$ (see Figure 3 ). However, the star is also too faint (by $0.16 \mathrm{mag}$ ) to meet the brightness cutoff for the star veto catalog of our pipeline, and hence was not removed by the 20.5 mag persistent-point-source cut in Section 2.

\section{Discussion}

Although our search identified two interesting candidates, it is unlikely that either candidate is associated with GW170814. Neither candidate is located in the $90 \%$ confidence region of the LALInference sky map, and both are associated with existing objects in DES catalogs that are inconsistent with our expectations of the GW source. Candidate 2 is likely the transient behavior of a variable star and is consistent with the number of background candidates expected in the blinded sample. Candidate 1 could also be stellar variability, or it could be a signal associated with a distant galaxy. Assuming that it is a galaxy, the DNF photometric redshift of the object is $z=0.95 \pm 0.12$, far beyond the possible redshift of GW170814 at that sky position. The 99\% upper limit on the GW distance along the Candidate 1 line of sight is $615 \mathrm{Mpc}$, whereas the galaxy distance is $6380_{-980}^{+1010} \mathrm{Mpc}$ assuming the LCDM cosmology parameters of Planck Collaboration (2016). 
We note, however, that photometric redshifts can occasionally have catastrophic failures.

An alternative explanation for the persistent emission from the two candidates is that one or both of these candidates is associated with a quasar. If either is a quasar, it is unlikely to be at the low redshifts of interest for GW170814 (Pâris et al. 2017). Spectroscopic follow up of the persistent sources associated with Candidates 1 and 2 could resolve the question of whether or not we have miscategorized them.

We conclude that these two candidates are not associated with GW170814, and thus we find no EM counterpart associated with the BBH merger over the $225 \mathrm{deg}^{2}$ region that we surveyed with $86 \%$ sky map coverage. We have not yet computed the efficiency, which is needed to set rate limits on $\mathrm{BBH}$ merger emission, but this rate-limit analysis is underway using SNANA simulations similar to those used in SoaresSantos et al. (2016). Our rate-limit analysis will also re-evaluate the cuts to maximize possible BBH model efficiency while minimizing SN background events. Qualitatively though, the analysis presented here covers $86 \%$ of the GW sky map and searches for events with rapidly declining light curves. The non-detection of an EM counterpart in our sample results in stringent limits on fast-declining optical models brighter than $i \sim 23$ mag 1-2 days after the BBH coalescence. This search is not sensitive to models that fade faster than the time between the first two observations due to Cut 4. Assuming a flat-infrequency optical spectrum from 4000 to $7000 \AA$ and the GW170814 median distance, this $i \sim 23$ mag limit corresponds to a luminosity limit of $L_{\text {optical }} \sim 5 \times 10^{41} \mathrm{erg} \mathrm{s}^{-1}$.

Our results constrain the space of models put forth in e.g., Stone et al. (2017) and de Mink \& King (2017). For example, Stone et al. (2017) posited that BBH mergers occurring in the gaseous environments of active galactic nuclei disks could be accompanied by gas accretion onto the final merged black hole that powers luminosities of order $L \sim 10^{40} \mathrm{erg} \mathrm{s}^{-1}$ lasting a few years, but highly super-Eddington accretion might result in a brighter and shorter-lived transient than our analysis is sensitive to. Our search also narrows the feasibility of models from de Mink \& King (2017), which predict emission with luminosities of approximately $L \sim 10^{42} \mathrm{erg} \mathrm{s}^{-1}$ occurring on fast timescales. The search performed here is tailored to remove longer-lived transients, and therefore it does not constrain longlived $\mathrm{BBH}$ counterparts, such as the $\mathrm{SN}$ association suggested in Loeb (2016) and later discussed in Woosley (2016) and D'Orazio \& Loeb (2018).

Aside from identifying interesting candidates, our search for counterparts to GW170814 is a test-bed for future BBH followup analyses where the sky-map credible areas will be small enough to be completely tiled in less than one night using DECam. For a real-time search for future counterparts, we consider resources to spectroscopically follow $\sim 10$ candidates, which we would want to identify within roughly two days of the GW trigger. In this scenario, we only apply the first five cuts in Table 2, as the remaining cuts depend on observations beyond two days. Through cut 5 ( $>2 \sigma$ decline), our search finds 45 candidates. Of these, we find that four candidates (including Candidates 1 and 2) are associated with DES-catalog objects that are either galaxies beyond our redshift cut (cut 3), or stars, and are thus uninteresting as black-hole-merger counterparts. Excluding these four candidates, our real-time search would find 41 candidates over $225 \mathrm{deg}^{2}$ or $\sim 16$ candidates per $87 \mathrm{deg}^{2}$ (the $90 \%$ credible area of the final
GW170814 sky map). For comparison, Cowperthwaite \& Berger (2015) predicted $\sim 19$ Type Ia SNe detected at $z<0.25$ over a 7 day, $87 \mathrm{deg}^{2}$ search. This suggests that the first five cuts are adequate to find interesting spectroscopic targets over a region the size of the GW170814 sky map.

Future work will incorporate simulations of $\mathrm{BBH}$ and $\mathrm{SN}$ light curves to assess the efficiency and false alarm rate of our search. If several BBH events are followed up with no EM counterpart found, a combined analysis will be needed to set limits on BBH EM emission.

\section{Conclusion}

We have presented an optical search for counterparts to gravitational wave GW170814 using DECam. Our search covered $225 \mathrm{deg}^{2}$, corresponding to $86 \%$ of the final LIGOVirgo sky map. Our difference-imaging pipeline produces 59560 light curves from the search images that are analyzed with the criteria in Section 3.2. After applying these cuts to the $i$-band light curves, two candidates remain. These two candidates are most likely not associated with GW170814: one is a high-confidence variable star, and the other is either a variable star or a transient associated with a high-redshift galaxy well beyond the expected GW170814 redshift.

With no candidates associated with GW170814, our analysis disfavors fast-declining optical emission from BBH mergers 1-2 days after merger with $i \lesssim 23$ mag. Future work will assess the efficiency and false-positive rate in optical BBH searches such as this one using simulations of BBH and SN light curves. Additionally, we will consider updates to our star veto catalog and galaxy catalog to account for fainter stars and objects with uncertain star or galaxy classification.

Tens of BBH signals are expected in the LVC's third operating run, and some are likely to have localization regions of similar size to that of GW170814. Based on the search and analysis presented here, we are preparing to search for additional BBH merger signals and quickly identify candidates for spectroscopic follow up. With future BBH optical searches and forward modeling of background and foreground signals, we will set increasingly stringent limits on BBH EM emission. Although BBH mergers may remain electromagnetically dark, the future of $\mathrm{BBH}$ astrophysics is bright.

Z.D. would like to thank Reed Essick for useful discussions and would also like to thank the Dark Cosmology Center and Neils Bohr Institute, where he was studying at the time of the GW170814 alert. Z.D. is supported by NSF Graduate Research Fellowship grant DGE-1144082. Z.D. and D.E.H. were partially supported by NSF CAREER grant PHY1151836 and NSF grant PHY-1708081. H.-Y.C. was supported by the Black Hole Initiative at Harvard University, through a grant from the John Templeton Foundation. R.J.F. is supported in part by NASA grant NNG17PX03C, NSF grant AST-1518052, the Gordon \& Betty Moore Foundation, the Heising-Simons Foundation, and by a fellowship from the David and Lucile Packard Foundation. This work was completed in part with resources provided by the University of Chicago Research Computing Center and support from the Kavli Institute for Cosmological Physics at the University of Chicago through NSF grant PHY-1125897 and an endowment from the Kavli Foundation.

Funding for the DES Projects has been provided by the DOE and NSF (USA), MEC/MICINN/MINECO (Spain), STFC 
(UK), HEFCE (UK). NCSA (UIUC), KICP (U. Chicago), CCAPP (Ohio State), MIFPA (Texas A\&M), CNPQ, FAPERJ, FINEP (Brazil), DFG (Germany), and the Collaborating Institutions in the Dark Energy Survey.

The Collaborating Institutions are Argonne Lab, UC Santa Cruz, University of Cambridge, CIEMAT-Madrid, University of Chicago, University College London, DES-Brazil Consortium, University of Edinburgh, ETH Zürich, Fermilab, University of Illinois, ICE (IEEC-CSIC), IFAE Barcelona, Lawrence Berkeley Lab, LMU München and the associated Excellence Cluster universe, University of Michigan, NOAO, University of Nottingham, Ohio State University, University of Pennsylvania, University of Portsmouth, SLAC National Lab, Stanford University, University of Sussex, Texas A\&M University, and the OzDES Membership Consortium.

Based in part on observations at Cerro Tololo InterAmerican Observatory, National Optical Astronomy Observatory, which is operated by the Association of Universities for Research in Astronomy (AURA) under a cooperative agreement with the National Science Foundation.

The DES Data Management System is supported by the NSF under grant No. AST-1138766 and AST-1536171. The DES participants from Spanish institutions are partially supported by MINECO under grants AYA2015-71825, ESP2015-66861, FPA2015-68048, SEV-2016-0588, SEV-2016-0597, and MDM-2015-0509, some of which include ERDF funds from the European Union. IFAE is partially funded by the CERCA program of the Generalitat de Catalunya. Research leading to these results has received funding from the European Research Council under the European Union's Seventh Framework Program (FP7/2007-2013) including ERC grant agreements 240672, 291329, and 306478. We acknowledge support from the Australian Research Council Centre of Excellence for Allsky Astrophysics (CAASTRO), through project number CE110001020, and the Brazilian Instituto Nacional de Ciência e Tecnologia (INCT) e-Universe (CNPq grant 465376/ 2014-2).

This manuscript has been authored by Fermi Research Alliance, LLC under contract No. DE-AC02-07CH11359 with the U.S. Department of Energy, Office of Science, Office of High Energy Physics.

\section{ORCID iDs}

Z. Doctor (1) https://orcid.org/0000-0002-2077-4914 R. Kessler (i) https://orcid.org/0000-0003-3221-0419 K. Herner (ㄷ) https://orcid.org/0000-0001-6718-2978

A. Palmese (1) https://orcid.org/0000-0002-6011-0530

M. Soares-Santos (i) https://orcid.org/0000-0001-6082-8529

J. Annis (i) https://orcid.org/0000-0002-0609-3987

D. Brout (1) https://orcid.org/0000-0001-5201-8374

D. E. Holz (1) https://orcid.org/0000-0002-0175-5064

A. Rest (1) https://orcid.org/0000-0002-4410-5387

P. Cowperthwaite (1) https://orcid.org/0000-0002-2478-6939

E. Berger (1) https://orcid.org/0000-0002-9392-9681

C. J. Conselice (1) https://orcid.org/0000-0003-1949-7638

M. S. S. Gill (1) https://orcid.org/0000-0003-2524-5154

R. E. Butler (1) https://orcid.org/0000-0003-2789-3817

H.-Y. Chen (i) https://orcid.org/0000-0001-5403-3762

R. Chornock (i) https://orcid.org/0000-0002-7706-5668

B. Farr (1) https://orcid.org/0000-0002-2916-9200

W. Fong (i) https://orcid.org/0000-0002-7374-935X

C. Fryer (ii) https://orcid.org/0000-0003-2624-0056
J. García-Bellido (i) https://orcid.org/0000-0002-9370-8360

R. Margutti (i) https://orcid.org/0000-0003-4768-7586

J. L. Marshall @i https://orcid.org/0000-0003-0710-9474

T. Matheson (1) https://orcid.org/0000-0001-6685-0479

B. D. Metzger (1) https://orcid.org/0000-0002-4670-7509

M. Nicholl (1) https://orcid.org/0000-0002-2555-3192

F. Paz-Chinchón (ib https://orcid.org/0000-0003-1339-2683

S. Salim (1i) https://orcid.org/0000-0003-2342-7501

L. F. Secco (1) https://orcid.org/0000-0002-6002-4288

N. Smith (1) https://orcid.org/0000-0001-5510-2424

A. K. Vivas (1) https://orcid.org/0000-0003-4341-6172

T. M. C. Abbott (1) https://orcid.org/0000-0003-1587-3931

D. Brooks (i) https://orcid.org/0000-0002-8458-5047

D. L. Burke (1) https://orcid.org/0000-0003-1866-1950

M. Carrasco Kind (1) https://orcid.org/0000-0002-4802-3194

F. J. Castander (1) https://orcid.org/0000-0001-7316-4573

S. Desai (1) https://orcid.org/0000-0002-0466-3288

D. W. Gerdes (i) https://orcid.org/0000-0001-6942-2736

D. A. Goldstein (1) https://orcid.org/0000-0003-3461-8661

D. Gruen (1) https://orcid.org/0000-0003-3270-7644

R. A. Gruendl (1) https://orcid.org/0000-0002-4588-6517

D. L. Hollowood (1) https://orcid.org/0000-0002-9369-4157

D. J. James (1) https://orcid.org/0000-0001-5160-4486

K. Kuehn (ㄷ) https://orcid.org/0000-0003-0120-0808

R. Miquel (1) https://orcid.org/0000-0002-6610-4836

B. Nord (i) https://orcid.org/0000-0001-6706-8972

R. L. C. Ogando (1) https://orcid.org/0000-0003-2120-1154

A. A. Plazas (ib https://orcid.org/0000-0002-2598-0514

A. Roodman (1) https://orcid.org/0000-0001-5326-3486

E. Sanchez (ib https://orcid.org/0000-0002-9646-8198

M. Smith (1) https://orcid.org/0000-0002-3321-1432

F. Sobreira (i) https://orcid.org/0000-0002-7822-0658

M. E. C. Swanson (1) https://orcid.org/0000-0002-1488-8552

G. Tarle (i) https://orcid.org/0000-0003-1704-0781

A. R. Walker (1) https://orcid.org/0000-0002-7123-8943

\section{References}

Abbott, B. P., Abbott, R., Abbott, T. D., et al. 2016a, PhRvL, 116, 061102 Abbott, B. P., Abbott, R., Abbott, T. D., et al. 2016b, ApJL, 818, L22 Abbott, B. P., Abbott, R., Abbott, T. D., et al. 2016c, PhRvL, 116, 241103 Abbott, B. P., Abbott, R., Abbott, T. D., et al. 2016d, PhRvX, 6, 041015 Abbott, B. P., Abbott, R., Abbott, T. D., et al. 2017a, PhRvL, 119, 161101 Abbott, B. P., Abbott, R., Abbott, T. D., et al. 2017b, PhRvL, 118, 221101 Abbott, B. P., Abbott, R., Abbott, T. D., et al. 2017c, ApJL, 851, L35 Abbott, B. P., Abbott, R., Abbott, T. D., et al. 2017d, PhRvL, 119, 141101 Arcavi, I., McCully, C., Hosseinzadeh, G., et al. 2017, ApJL, 848, L33 Bernstein, G. M., Abbott, T. M. C., Desai, S., et al. 2017, PASP, 129, 114502 Chen, H.-Y., Fishbach, M., \& Holz, D. E. 2018, Natur, 562, 545

Connaughton, V., Burns, E., Goldstein, A., et al. 2016, ApJL, 826, L6 Coulter, D. A., Foley, R. J., Kilpatrick, C. D., et al. 2017, Sci, 358, 1556 Cowperthwaite, P. S., \& Berger, E. 2015, ApJ, 814, 25

Cowperthwaite, P. S., Berger, E., Soares-Santos, M., et al. 2016, ApJL, 826, L29

de Mink, S. E., \& King, A. 2017, ApJL, 839, L7

De Vicente, J., Sánchez, E., \& Sevilla-Noarbe, I. 2016, MNRAS, 459, 3078 D'Orazio, D. J., \& Loeb, A. 2018, PhRvD, 97, 083008

Drlica-Wagner, A., Sevilla-Noarbe, I., Rykoff, E. S., et al. 2018, ApJS, 235, 33 Fishbach, M., Gray, R., Magaña, I., et al. 2019, ApJ, 871, L13 Flaugher, B., Diehl, H. T., Honscheid, K., et al. 2015, AJ, 150, 150 Goldstein, D. A., D'Andrea, C. B., Fischer, J. A., et al. 2015, AJ, 150, 82 Gupta, R. R., Kuhlmann, S., Kovacs, E., et al. 2016, AJ, 152, 154 Guy, J., Sullivan, M., Conley, A., et al. 2010, A\&A, 523, A7 Kessler, R., Bassett, B., Belov, P., et al. 2010, PASP, 122, 1415 Kessler, R., Bernstein, J. P., Cinabro, D., et al. 2009, PASP, 121, 1028 Kessler, R., Marriner, J., Childress, M., et al. 2015, AJ, 150, 172 LIGO-Virgo Collaboration 2017a, LIGO/Virgo G297595: Identification of a GW Binary Merger Candidate, https://gcn.gsfc.nasa.gov/other/G297595.gen3 
LIGO-Virgo Collaboration 2017b, LIGO/Virgo G297595: Updated Localization from LIGO and Virgo Data, https://gcn.gsfc.nasa.gov/other/G297595.gcn3

Lipunov, V. M., Kornilov, V., Gorbovskoy, E., et al. 2017, MNRAS, 465,3656

Loeb, A. 2016, ApJL, 819, L21

McKernan, B., Ford, K. E. S., Bellovary, J., et al. 2018, ApJ, 866, 66

Morganson, E., Gruendl, R. A., Menanteau, F., et al. 2018, PASP, 130, 074501

Morokuma, T., Tanaka, M., Asakura, Y., et al. 2016, PASJ, 68, L9

Neilsen, E. H., Jr., Bernstein, G., Gruendl, R., \& Kent, S. 2016, Office of Scientific and Technical Information, Technical Report FERMILAB-TM2610-AE-CD 1450750

Pâris, I., Petitjean, P., Ross, N. P., et al. 2017, A\&A, 597, A79

Perna, R., Lazzati, D., \& Giacomazzo, B. 2016, ApJL, 821, L18

Phinney, E. S. 2009, arXiv:0903.0098

Planck Collaboration 2016, A\&A, 594, A13

Schutz, B. F. 1986, Natur, 323, 310
Sevilla-Noarbe, I., Hoyle, B., Marchã, M. J., et al. 2018, MNRAS, 481, 5451 Singer, L. P., \& Price, L. R. 2016, PhRvD, 93, 024013

Smartt, S. J., Chambers, K. C., Smith, K. W., et al. 2016a, MNRAS, 462, 4094 Smartt, S. J., Chambers, K. C., Smith, K. W., et al. 2016b, ApJL, 827, L40

Smith, G. P., Bianconi, M., Jauzac, M., et al. 2018, arXiv:1805.07370

Soares-Santos, M., Holz, D. E., Annis, J., et al. 2017, ApJL, 848, L16

Soares-Santos, M., Kessler, R., Berger, E., et al. 2016, ApJL, 823, L33

Stalder, B., Tonry, J., Smartt, S. J., et al. 2017, ApJ, 850, 149

Stone, N. C., Metzger, B. D., \& Haiman, Z. 2017, MNRAS, 464, 946

The DES Collaboration, the LIGO Scientific Collaboration, the Virgo Collaboration et al. 2019, arXiv:1901.01540

The LIGO Scientific Collaboration, the Virgo Collaboration 2018, arXiv:1811. 12907

Valenti, S., David, K., Sand, J., et al. 2017, ApJL, 848, L24

Woosley, S. E. 2016, ApJL, 824, L10

Yoshida, M., Utsumi, Y., Tominaga, N., et al. 2017, PASJ, 69, 9 